\title{
Aspects méthodologiques et prospectifs du maintien et du rétablissement des voies de migration
}

\author{
Methodological and prospective \\ aspects relating to maintenance \\ and restoration of migration channels
}

\author{
M. Roguet \\ Conseil supérieur de la pêche \\ Toulouse
}

\author{
M. Larinier \\ CEMAGREF \\ Castanet Tolosan
}

Dans une première partie, on rappelle les composantes scientifiques et techniques des problèmes touchant aux dispositifs de franchissement. Les progrès de la technique dans le domaine, que ce soit pour assurer la migration vers l'amont ou l'aval, sont avant tout alimentés par l'expérimentation et le suivi des installations existantes.

Dans une deuxième partie, on évoque les problèmes plus généraux posés par la protection ou la restauration des stocks de poissons migrateurs.

In the first part the emphasis is placed on scientific and technical components of problems relating to the pass devices. The progress or the technique in this field, whether it is to assume migration upstream or downstream, is first and foremost supplied by experiment and the follow-up of existing installations.

The second part refers to more general problems posed by the protection and restoration of stocks of migrating fish.

1. Aspects et composantes techniques et scientifiques des problèmes touchant au franchissement des ouvrages.

Les progrès techniques dans le domaine de la conception des ouvrages de franchissement sont avant tout assurés par l'expérimentation : études in-situ ou sur modèle réduit en laboratoire, suivi des installations existantes (contrôle, comportement du poisson, améliorations progressives). Les pays de référence dans le domaine sont ceux ayant le plus expérimenté et dans lesquels la conception et l'efficacité des passes relevaient de la responsabilité des maîtres d'ouvrage.

Depuis une dizaine d'années en France, un gros effort a été entrepris dans l'aménagement de dispositifs de franchissement. La plupart de ces passes ont été installées sur des cours d'eau de petite et moyenne importance (plus d'une centaine en Bretagne et Basse Normandie) et concernent avant tout les salmonidés migrateurs. L'expérience acquise sur les grands axes (Dordogne, Garonne, Loire...) reste encore très limitée. 


\subsection{Suivi des ouvrages}

Il conviendrait dans les prochaines années de développer des opérations de contrôle systématique d'un certain nombe de dispositifs de franchissement présentant un intérêt particulier :

- soit par leur conception originale

- soit par l'importance que revêt leur efficacité pour certains programmes de restauration

- soit parce que leur efficacité pour des espèces migratrices particulières : (alose, brochet, ombre) est mal connue.

On citera pour mémoire, l'importance du suivi des ouvrages de franchissement de Bergerac sur la Dordogne, de Golfech sur la Garonne, de Bec des deux eaux sur l'axe Vienne-Creuse-Gartempe, de Vichy et Poutès sur l'Allier.

Les écluses à poissons, en particulier celles récemment installées sur le Tarn, le Lot, le Gave de Pau, le Guiers et le Haut Rhône devraient faire l'objet d'un contrôle systématique.

On cherche à affiner les limites de fonctionnement (espèces et tailles en fonction des conditions hydrauliques et thermiques) des passes à ralentisseurs les plus couramment utilisées.

\subsection{Techniques d'évaluation de l'efficacité des passes}

Si les opérations de piégeage à la montée sont souvent relativement simples à mettre en œuvre, après une phase de mise au point inévitable, la gestion et la maintenance de l'outil se révèlent très contraignantes, le facteur humain étant déterminant (nécessité de la présence quasi permanente d'un ou plusieurs opérateurs).

Les efforts devront porter sur la mise au point de techniques moins lourdes, telles qu'utilisation de compteurs automatiques, recours aux techniques vidéo, échantillonnages, etc. Les recherches et expérimentations dans le domaine sont en France très récentes et encore trop peu nombreuses. Si plusieurs compteurs à résistivité ont été installés, on est loin de connaitre leurs conditions et limites d'utilisation ainsi que leur fiabilité.

La mise au point de tels outils permettra de mieux connaître l'importance les rythmes saisonniers et journaliers des migrations ainsi que les effets des paramètres du milieu sur ces migrations, autant d'éléments indispensables à une gestion efficace des stocks.

\subsection{Techniques de radio-pistage}

Plusieurs équipes opérationnelles dans le domaine du radio-pistage devront être formées au sein d'organismes scientifiques ou techniques de façon à faire progresser la connaissance du comportement migratoire des différentes espèces, connaissances susceptibles de permettre l'amélioration des conditions de franchissement de plusieurs obstacles sur les grands axes (obstacles sur la Loire, Vichy, etc.).

\subsection{Expérimentations dans le domaine de la dévalaison}

Les problèmes de franchissement par les poissons des obstacles à la descente, qu'il s'agisse des juvéniles, des adultes ayant survécu à la reproduction (saumons, truites de mer) ou des anguilles ont été sous-estimés dans le passé. Des programmes devront être envisagés pour définir un certain nombre de dispositifs efficaces et réalistes permettant d'empêcher ou tout au moins de réduire significativement l'entraînement des poissons dans les turbines.

\subsection{Stages de formation}

Il est essentiel pour la poursuite des programmes de restauration que l'information concernant l'expérience acquise dans le domaine soit centralisée, synthétisée et fasse l'objet d'une large diffusion. Des stages de formation, organisés depuis 5 ans par le C.S.P. et le C.E.M.A.G.R.E.F., avec la collaboration d'E.D.F. et destinés dans un premier temps au personnel du C.S.P. sont maintenant ouverts aux administrations, aux maîtres d'œuvre, aux bureaux d'étude à E.D.F. ainsi qu'aux grandes compagnies d'aménagement ou entreprises de travaux publics.

\subsection{Organisation dans le domaine de la conception et du contrôle des passes à poissons.}

Les maîtres d'ouvrage restent responsables des conséquences induites par leurs interventions sur les cours d'eau, notamment en ce qui concerne le maintien des stocks de poissons migrateurs, et assujettis à une obligation de résultat. Comme on l'a noté plus haut, c'est dans les pays où ce principe a été mis en pratique de façon rigoureuse qu'ont été réalisés les progrès les plus significatifs dans le domaine.

Il appartient cependant aux pouvoirs publics de contrôler le sérieux des projets ainsi que leur bonne réalisation, ceci afin d'éviter dans la mesure du possible les surcoûts résultant d'installations trop mal conçues.

L'Administration ou les techniciens amenés à formuler un avis sur un projet de passe à poissons ne pourront le faire efficacement que dans la mesure où la consistance du dossier présenté le leur permettra, dossier devant inclure obligatoirement un certain nombre d'éléments, parmi lesquels :

- des informations sur les espèces migratrices, les caractéristiques hydrologiques et thermiques du cours d'eau; les plans du site et la topographie à l'aval de l'obstacle, les caractéristiques de tous les ouvrages évacuateurs, des turbines, le mode d'exploitation de l'ouvrage;

- les niveaux d'eau amont et aval à considérer en période de migration;

- les plans détaillés du dispositif de franchissement avec une note de calcul précisant le comportement hydraulique de la passe pour les niveaux d'eau amont et aval en période de migration.

L'attention des maîtres d'ouvrage devrait être explicitement attirée sur leur obligation de résultat, en particulier sur le fait que des modifications peuvent être demandées au dispositif initial si son efficacité ne se révèle pas satisfaisante après quelques années. L'agrément d'experts en matière de conception de passes à poissons devrait être envisagé. 


\section{Problèmes généraux posés par le maintien et la restauration des populations d'espèces migratrices.}

\subsection{Information et sensibilisation}

La première recommandation formulée en conclusion du Colloque franco-québecois sur la restauration des rivières à saumons (Bergerac, 1985) a été l'urgence d'une large information rappelant la nécessité du maintien et du rétablissement des voies de migration :

- auprès des pêcheurs en mer sur les zones d'engraissement dans la zone littorale, en estuaire et en rivière et parmi ces derniers, aux pêcheurs aux engins ou à la ligne qui souhaitent souvent voir les poissons arrêtés ou ralentis par les obstacles;

- auprès des aménagistes construisant barrages et seuils sur les cours d'eau;

- auprès des politiques et des élus locaux;

- auprès des administrations chargées de la police de l'eau et de la pêche.

L'information doit notamment porter sur le rôle, l'efficacité et les limites des passes à poissons; si la technologie a fait en France au cours de ces dernières années des progrès notables, ceux-ci pourront être rapprochés du développement de la chirurgie réparatrice ou orthopédique dont les progrès naissent des grands conflits et de l'automobile. La meilleure prothèse ne vaudra cependant jamais le membre qu'elle remplace. Il faut donc se garder avec soin des deux attitudes extrêmes suivantes :

- celle des pessismistes encore largement répandue bien qu'en régression consistant à dire que les passes à poissons n'ont jamais fonctionné en s'appuyant sur des références historiques qui n'ont plus rien à voir avec la réalité d'aujourd'hui;

- celle des optimistes invétérés qui pensent - ou ont intérêt à penser - que la technique peut faire tous les miracles et seraient prêts à barrer tous les cours d'eau à migrateurs.

La sensibilisation du public est également un point important. Les salles de visites permettant de voir passer les migrateurs dans la partie amont des passes, se révèlent être des outils de sensibilisation très efficaces $(300000$ visiteurs au barrage de Bonneville (USA), intérêt du public pour le suivi de la passe de Bergerac...).

\subsection{Programme de restauration}

\subsubsection{Montage des programmes de restauration}

L'une des conclusions du Colloque de Bergerac est que toute opération de restauration doit faire l'objet d'un programme à moyen et long terme, réclamant impérativement des actions coordonnées conduites avec rigueur et cohérence dans un consensus général. La tâche est particulièrement difficile en France où les responsabilités relatives à la pêche, au contrôle des pollutions, à l'utilisation agricole ou énergétique de l'eau sont réparties entre de nombreux services administratifs, établissements publics et organisations associatives.
Tout lancement d'un programme de restauration doit être précédé d'une étude de faisabilité portant sur :

- les potentialités du cours d'eau, basées sur sa capacité d'accueil, fonction de la surface et de la qualité de ses frayères et des secteurs de grossissement des juvéniles; - l'inventaire complet des obstacles à la migration, tant à la montée qu'à la descente comprenant une évaluation technique, une estimation approximative du coût des dispositifs nécessaires ainsi qu'une analyse juridique basée sur l'examen des droits d'eau;

- la définition d'une politique de soutien des effectifs (saumons, truites de mer) à partir d'installations existantes ou à créer;

- la définition des travaux nécessaires pour améliorer le milieu aquatique (lutte contre la pollution, amélioration des zones de frayères et de grossissement).

Cette étude propose un calendrier des réalisations à entreprendre par ordre d'urgence et indique le montant approximatif du coût du programme. Le projet ainsi établi doit être présenté aux divers intervenants (État, Région, Département, Comités ou Associations de riverains, établissements publics, etc.) pour adoption et financement éventuel.

\subsubsection{Réalisation}

La réalisation de ces programmes se traduira :

- par des mesures de protection du milieu sur l'ensemble du cours d'eau, voire du bassin (rivières réservées, arrêtés de biotope);

- par le lancement d'interventions (aménagement de dispositifs de franchissement, amélioration de la qualité de l'eau et de l'habitat,

- par le suivi biologique des populations et la quantification de l'incidence des divers aménagements sur ces populations (efficacité des passes, retards à la migration des adultes à l'aval des obstacles, mortalités des juvéniles dans les turbines, prédation...);

- par la prise de décisions nécessaires à la gestion à long terme des stocks des espèces migratrices (obligation de la déclaration et du marquage de tous les sujets capturés, par quelque mode que ce soit pour le saumon);

- par un certain nombre d'actions complémentaires comme la recherche de souches les mieux adaptées au cours d'eau, le transport provisoire de géniteurs à partir d'un ouvrage équipé d'un piège soit sur les frayères, soit en pisciculture;

- par des repeuplements, au moins jusqu'à obtention d'un stock suffisant de géniteurs de retour.

La réussite d'un programme de restauration implique qu'aucune des actions précitées ne soit oubliée. Il doit aboutir au bout d'une dizaine d'années à des résultats tangibles, faute de quoi certaines mesures de protection du milieu peuvent apparaître de moins en moins supportables et être remises en question.

\subsubsection{Les moyens nécessaires}

La définition et la réalisation des programmes de restauration nécessitent des moyens juridiques, administratifs, financiers, techniques et scientifiques.

Les moyens juridiques indispensables pour assurer la protection du milieu et pour permettre la réalisation des 
ouvrages nécessaires existent (police de l'eau, loi sur la protection de la Nature, sur la production d'énergie hydroélectrique, nouvelle loi sur la pêche). Leur mise en œuvre demande une volonté et des moyens en personnels.

Si l'Etat lui-même a lancé les programmes de restauration, il faut pour chacun un meneur de jeu qui peut être selon le cas, une Administration (DDAF, DRAE, DDE), une Délégation régionale du Conseil supérieur de la pêche, une Fédération de pêche, voire une Association.

Il est également nécessaire que les grands organismes aménageurs (E.D.F.; compagnies d'aménagement) s'impliquent largement dans les programmes.

Pour financer la réalisation des programmes de restauration, (Dordogne, Adour, Garonne, Gartempe, axe Loire-Allier), des contrats Etat-Région ont été conclus dans plusieurs régions (Aquitaine, Midi-Pyrénées Limousin). Aux financements de l'Etat et de la Région peuvent s'ajouter ceux du département, des pêcheurs (CSP + Fédérations ou Pêcheurs professionnels). La passe à poissons du Ramier à Toulouse sera financée conjointement par le propriétaire (47\%), l'Etat (11\%), la Région $(11 \%)$, le Conseil général (22\%), le Conseil supérieur de la pêche $(9 \%)$.

Dans le cas de la mise en chantier d'un contrat de rivière, le programme de restauration doit être inclus dans les actions inscrites au contrat qui ne peuvent qu'aider à l'aboutissement du projet (contrats de rivière Vézère, Aveyron...).

Le principe retenu en Midi-Pyrénées et Aquitaine est qu'une subvention ne peut être accordée que pour un aménagement en situation juridique régulière, ce qui exclut tout aide de ce type pour la réalisation de dispositifs imposés par l'autorisation ou la concession; il peut s'agir soit de la réfection de dispositifs inefficaces, soit de leur réalisation en dehors de toute obligation.

L'article 411 impose au propriétaire, la réalisation sans indemnité de dispositifs sur les cours d'eau classés, dans les 5 ans suivit la parution de la liste des espèces migratrices. Ceci ne parait pas exclure des formes de financement telles que les prêts dont le remboursement serait garanti, dans le cas de microcentrales, par la production d'électricité : modèle pourrait être pris sur le Fond forestier national qui, alimenté par une taxe sur les bois, accorde des subventions et des prêts remboursables pour certains sur le produit des premières coupes.

Les opérations de restauration et de gestion sont avant tout expérimentales et exigent des moyens en personnel importants: biologistes pour assurer une meilleure connaissance du milieu et le suivi des populations pour proposer des plans de gestion; techniciens pour la conception, le suivi et la valorisation des ouvrages; personnels chargés de la police de l'eau et de la pêche.

Ces moyens sont manifestement insuffisants à l'heure actuelle et ne sont pas à la mesure de l'ambition des programmes de restauration, en particulier sur les grands axes.

\subsection{Protection des cours d'eau ne faisant pas l'objet d'un programme de restauration}

Le problème de l'installation d'une passe à poissons à l'occasion de la construction d'un barrage ou de la modification d'un ouvrage existant peut se poser sur un cours d'eau ne faisant pas l'objet d'un plan de restauration.

La nature de la décision sera fonction de plusieurs éléments qui devraient être clairement exprimés dans l'étude ou la notice d'impact.

- si le cours d'eau est un ancien axe de migration non inclus dans un programme de restauration du fait de difficultés techniques actuelles mais qu'il subsiste un habitat favorable (zones à juvéniles, frayères potentielles) il paraît impératif de prévoir un dispositif de franchissement (ex: le Lot);

- si le cours d'eau comporte des espèces holobiotiques dont la migration est indispensable au déroulement du cycle biologique (brochet, truite, ombre), la passe est nécessaire;

- la pollution ne doit jamais être considérée comme une condamnation définitive d'un cours d'eau (ex. la Dore) et ne saurait être un obstacle à la construction d'une passe.

\section{Conclusion}

A de nombreuses reprises au cours de l'histoire, des tentatives ont été faites pour rendre à certains cours d'eau leurs migrateurs.

Ces tentatives, cycliques, se sont traduites par des échecs pouvant s'expliquer pour les raisons suivantes :

- moyens d'action insuffisants au plan juridique ne permettant pas de régler tous les problèmes présents sur un axe de migration;

- pas de consensus politique, tentatives restées confidentielles;

- coordination administrative insuffisante;

- pas de moyens techniques adéquats (passes inefficaces, mettant un terme aux programmes, technique d'élevage et d'alevinage des jeunes saumons pas au point);

- programmes manquant de cohérence (repeuplements sans création de dispositifs de franchissement, construction de passes sans protection de biotope...);

- indifférence générale à l'égard des problèmes d'environnement dans les périodes de reconstruction ou d'expansion avec de grands besoins énergétiques.

Il faut espérer que, tirant des leçons du passé, notre époque pourra s'enorgueillir d'avoir su maintenir sur les axes encore fréquentés et rétablir sur certains de nos cours d'eau l'immense richesse que constituent les grands migrateurs.

\section{Discussion}

Président : M. J.C. VINÇONNEAU

M. Le Président. - Mesdames, Messieurs, nous abordons la dernière ligne droite de cette session, et je dois vous féliciter de votre assiduité. J'ai même l'impression que vos rangs se sont encore resserrés depuis le début de la session car d'autres personnes nous ont rejoints. Je voudrais aussi vous remercier pour votre discipline car nous commençons, à la minute près, cette partie de notre session. 
En accord avec le Président Cazenave, nous avons voulu que cet après-midi soit consacrée à la fois à la synthèse de nos différents travaux, à une partie, je dirai, "grand public " qui va être le film; puis à une table ronde qui nous permettra de faire le point, de poser les dernières questions et de tirer des conclusions pour les travaux ultérieurs aussi bien de la Société Hydrotechnique de France, de l'Agence de bassin et des différents Organismes que vous représentez.

Je suis chargé donc de présider la première partie, c'est-à-dire les deux exposés de synthèse, l'un sur l'aspect juridique, l'autre sur l'aspect technique. Chaque exposé durera une demi-heure, ensuite nous avons le film.

Si vous êtes d'accord sur cette façon de procéder, je donne immédiatement la parole à $M$. Roguet, Délégué régional du Conseil supérieur de la Pêche, qui, en collaboration avec $\mathrm{M}$. Larinier, du CEMAGREF, en résidence à Toulouse, va nous exposer :

Les aspects méthodologiques et prospectifs du maintien et du rétablissement des voies de migration

\section{Exposé}

M. Le Président. - Comme président de séance, jé dois remercier et féliciter M. Roguet à la fois pour le contenu et le brio de son exposé; je le féliciterai aussi pour le fait qu'il ait respecté scrupuleusement l'horaire.

Je crois que son exposé répond bien à ce qu'on avait demandé, c'est-à-dire faire une synthèse sur nos travaux et préparer une ouverture sur d'autres problèmes.

Avez-vous des questions à poser à M. ROGUet et M. LARINIER ?...

M. TANE. - Je voudrais demander à M. ROGUET s'il peut expliquer rapidement sous quelle forme il voit un fonds de restauration...

De mon côté j'avais fait remarquer à plusieurs personnes qu'il y a un aspect qui doit être réglé : c'est l'aspect «article 410 du Code rural... La question va peut-être être traitée par M. DENOZIERE...

On avait pensé à une taxe sur les micro-centrales, mais je crains que tout le monde ne soit pas d'accord.

M. ROGUET. - Les fabricants d'électricité nous ont toujours dit qu'ils travaillaient pour la collectivité nationale, et pas tellement pour eux. Or il y a des périodes dans l'année où le courant électrique n'est pas très utile : c'est l'été; et à ces moments-là au contraire l'eau est très utile.

Alors j'avais dit: puisque ces messieurs travaillent pour la collectivité de façon désintéressée, quand ils font de l'électricité d'été, au lieu de virer cela à leur compte, on pourrait le virer à un compte destiné à l'environnement... Mais je pense que ma proposition risque de provoquer des réactions houleuses...

M. MORELLO. - La contribution des micro-centrales au budget de l'Etat se chiffre par centaines de millions de centimes. Donc la réponse est toute trouvée. faut !

M. ROGUET. - Ce ne sont peut-être pas des centimes qu'il nous

M. Morello. - Si ce n'est pas ce qu'il vous faut, alors...

M. ROGUET. - Sur le plan des besoins en argent il faut quand même examiner ce que cela peut représenter.

Sur le gave d'Oloron, la Nive, et les affluents,... il y aurait 35 ouvrages à équiper sur 103 ... Si l'on compte 500000 francs par ouvrage en moyenne (c'est peut-être même beaucoup), cela ferait quelques 17,5 millions de francs... Je dis bien que c'est une première estimation.

Par comparaison, disons qu'avec $50 \mathrm{~m}$, ou même $20 \mathrm{~m}$ du pont de Cahors, qui va permettre aux vacanciers de gagner 10 minutes en été, aux heures de pointe, on réglerait complètement le problème.
On nous dit que la passe à poissons de Golfech va coûter cher. C'est vrai... Peut-être jusqu'à 15 millions de francs... En admettant même qu'elle aille jusqu'à 20 millions, cela correspond à $0,5 \%$ de deux tranches nucléaires... Alors il faut relativiser les coûts.

M. MORELLO. - La contribution des micro-centrales privées est de 120 millions de francs actuels, par an.

M. PICARD, - C'est une question à voir sur le plan économique et financier. Chaque secteur, dans son domaine, considère toujours que finalement le volume financier qu'il met en jeu est faible par rapport à d'autres.

A mon avis, c'est un problème de choix politique que de déterminer quelle somme on souhaite consacrer à ce type d'action.

Je crois qu'on peut faire confiance aux Services de M. TANE pour défendre le dossier et obtenir le maximum de crédits, soit directement, soit par des mesures consistant à faire payer un certain nombre d'Organismes... parce qu'il y en a qui Profitent (avec un grand $\mathrm{P}$ ), je n'hésite pas à le dire, des richesses naturelles. Les promoteurs des micro-centrales s'ils versent beaucoup de taxes à l'Etat et aux collectivités ne sont pas des philanthropes.

Pour ce qui est de la deuxième question je ne suis pas sûr d'avoir bien compris... Il me semble que $M$. Roguet a dit que l'orsqu'on travaille sur un axe il faut tout faire, et que si on laissait de côté un des éléments c'était comme si l'on ne faisait rien.

Cette réflexion m'inquiète. Est-ce qu'on ne peut pas, à propos d'un axe, déterminer les priorités, les points les plus sensibles, sachant que dans une deuxième ou une troisième étape on obtiendra plus.

Si l'on ne peut pas agir de cette manière, on sera inévitablement conduit à limiter le nombre d'axes.

M. ROGUET. - Je crois avoir dit qu'il fallait faire des programmes cohérents, mais, si l'on veut, des tranches fonctionnelles.

Pour le programme présenté à la région Midi-Pyrénées, il y a une tranche 1984/1988 qui va s'arrêter au barrage de La Barre. On estime que de cette manière on pourra régler les 5 ou 6 problèmes qui se posent... Il faut « mettre le paquet " sur quelques problèmes et ne pas en démordre... Après cela, on passera à la tranche suivante.

Mais une partie des indications que j'ai données doit trouver une solution immédiate : il faut régler la question de la protection de l'axe en précisant ce qui peut être fait en totalité et jusqu'où l'on peut aller dans une première étape.

C'est important parce que si pendant qu'on fait la première tranche on compromet la deuxième par certains ouvrages ou par des extractions de graviers, on aura raté une grande partie de l'opération. C'est pour cela que le consensus doit porter sur la totalité du programme, avec des tranches fonctionnelles de restauration à l'intérieur desquelles il faut attaquer les choses dans l'ordre d'urgence.

Par ailleurs il y a les aspects juridiques : il y a des cas sur lesquels on bute de ce point de vue.

C'est pourquoi les mesures financières incitatives sont intéressantes.

C'est ainsi que sur l'Ariège il y a 4 barrages, dont les autorisations expirent en 1994. On a pu négocier avec le propriétaire en lui disant : si vous demandez immédiatement des subventions, on vous les donnera; si vous attendez 1994, il faudra que vous fassiez les travaux on ne vous donnera plus rien. Eh bien ! l'intéressé a demandé des subventions.

M. Le Président. - Je voudrais remercier MM. Roguet et LARINIER pour leur contribution à cet exposé, et, à travers eux, le Conseil supérieur de la Pêche et le CEMAGREF qui nous ont aidés par leur intervention et leur appui technique à organiser cette session. Je remercie les deux directeurs qui sont présents. 
I.U.T.A.M.

UNION INTERNATIONALE DE MÉCANIQUE THÉORIQUE ET APPLIQUÉE

ANNONCE PRÉLIMINAIRE

\section{$17^{\text {e }}$ Congrès International de Mécanique Théorique et Appliquée}

Institut de Mécanique de Grenoble

GRENOBLE $\cdot 21-27$ août 1988

Le $17^{\text {e }}$ Congrès se tiendra à GRENOBLE - SAINT-MARTIN-D'HĖRES aux dates précitées. II couvrira l'ensemble des Sciences mécaniques, mécanique analytique, mécanique des solides et des fluides, y compris les applications. Une quinzaine de conférenciers sont invités par le Comité des Congrès; le nombre des autres communications, qui seront faites oralement ou sur panneaux pourra atteindre 480 .

Dans le cadre du congrès seront organisés trois colloques spécialisés :

Mécanique des grandes déformations et de l'endommagement des matériaux.

Dynamique des écoulements diphasiques.

Mécanique de la croûte terrestre.

La mécanique des fluides et la mécanique des solides, ainsi que le génie mécanique sont concernés par les trois sujets choisis. Lors de la première demi-journée de chacun de ces colloques spécialisés, des conférences d'ouverture présenteront les aspects généraux du sujet.

Les participants souhaitant présenter une communication doivent envoyer six exemplaires d'un bref résumé de 100 à 150 mots, et six exemplaires d'un texte plus détaillé (de l'ordre de 500 mots); ils doivent également faire parvenir des copies de leurs diapositives dont la qualité peut être prise en compte lors de la sélection. L'envoi doit être fait au Secrétariat du Congrès, la date limite étant le 8 février 1988. Pour faciliter le choix du Comité, il est souhaitable que les auteurs précisent le mode de présentation préféré, oral ou sur panneaux, la décision finale restant évidemment au Comité.

Des annonces plus détaillées paraitront en 1987. Les personnes intéressées par ces annonces sont priées de l'indiquer en écrivant au Secrétariat du Congrès, à l'adresse suivante :

Pr. D. CAILLERIE

Secrétaire de I'ICTAM 1988

Institut de Mécanique de Grenoble - Domaine Universitaire - B.P. 68

38402 SAINT-MARTIN-D'HËRES CEDEX - France 